Nitroxide of Amylen.-Discovered by the exhibitor. of historical interest as being the first instance in which nitroxyl $\mathrm{NO}_{2}$ was shown to behave as a balogen in uniting directly with an olefine to form a body homologous with "Dutch liquid." The composition of the body is $\mathrm{C}_{5} \mathrm{H}_{50}\left(\mathrm{NO}_{2}\right)_{2}$.

Sulphide of Enanthyl-Discovered by the exhibitor, and of historical interest as being the first instance in which a term of a higher alcohol series was made from terms of lower alcohols. It is formed by the action of zinc ethyl on sulpho-chloride of amylen.

And Nitrate of Amyl.-Discovered by M. Balard. Its therapeutic action was discovered, and its introduction into the pharmacopœia recommended, by the exhibitor; and it is now coming into use in tetanic and other nervous affections.

A series of twenty-three specimens of hydrocarbons derived from Pennsylvanian petroleum is exhibited by Prof. Schorlemmer. They form a striking record of the skill with which a most laborious and difficult investigation has been conducted.

Very interesting and important are the ethyl compounds derived from the isolated radical methyl exhibited by $\mathrm{Mr}$. W. H. Darling. The results of some experiments made by myself seemed to indicate that the products of the action of chlorine upon methyl were not ethyl compounds; but the experiments of Schorlemmer and Darling conducted with much larger quantities of material, show that my conclusion was erroneous. Mr. Darling exhibits ethylic chloride, ethylic alcohol, ethylidenic chloride and sodic acetate, all made from electrolytic methyl.

Mr. Perkin has sent a large collection of specimens illustrating his researches on mauveine, artificial alizarin, artificial coumarin, glyoxylic acid, and other subjects. His investigation of glyoxylic acid seems to have at last put an end to the controversy as to the possibility of two semimolecules of hydroxyl being united with one and the same atom of carbon. I will not, however, anticipate Mr.Perkin, who will, I trust, personally give us an account of his researches.

Amongst the other exhibits in this department are numerous and important contributions from the laboratories of St. Petersburg, Louvain and Edinburgh. For several years past chemical research has been actively carried on in Russia.

The apparatus used in Kesearch exhibited in the Chemical Section has suffered much from the depredations of the physicists, for although chemistry is essentially founded upon measurements of weight and volume, the instruments used for such determinations have been swept almost en masse into the section of meäsurement; nevertheless, the chemical section contains several objects of unusual interest. The apparatus with which chemists, both ancient and modern, prosecuted their researches was generally of a simple description and often dismantled as soon as the necessary operations were completed, consequently it was far less likely to be preserved than the more expensive and elaborate contrivances of the physicist. Here, however, is Black's balance presented to the Science and Art Museum of Edinburgh, by the Right Hon. Lyon Playfair. Upon this balance Dr. Black ascertained in 1757 , the loss of wcight suffered by carbonate of magnesia and limestone when exposed to heat. Hales previously used a balance for this purpose, but the instrument before us was certainly one of the first employed for quantitative chemistry. The balances used by Cavendish, Davy, Young, and Dalton are here, and each one of them has its own historical interest for the chemist The balance of Cavendish is probably the instrument with which in 1783 or 1784 he first ascertained that a globe filled with a mixture of oxygen and hydrogen gases underwent no aiteration in weight when the mixture was exploded.

From gravimetric instruments we are naturally led to volumetric apparatus used in quantitative chemistry, and
I will now, in conclusion, briefly direct the attention of the conference to apparatus used in the analysis of gases, in the hope that a discussion of the merits and defects of the numerous instruments now before me may have the effect of directing a larger share of attention to eudiometric chemistry than has hitherto been accorded to it. This branch of chemical analysis originated in the attempts of Fontana, Landriani, Scheele, Priestley, Cavendish, Gay Lussac, Dalton, and others, to determine the volume of oxygen in samples of atmospheric air taken from various localities. In these primitive instruments air was exposed to the action of some substance either solid, liquid, or gaseous, which combined with the oxygen and left the nitrogen unacted upon. The chief substances used were phosphorus, potassic sulphide, nitric oxide, a solution of nitric oxide in ferrous sulphate, and a mixture of sulphur and iron filings. Many of the instruments were of simple or even rude construction, and little calculated to inspire confidence in the results. Nevertheless, the accuracy of a determination often depends much more upon the skill of the operator than upon the construction of the instrument used ; and thus Cavendish, with nitric oxide as his reagent and water as the confining liquid, made many hundred analyses of air, collected in various localities, in $178 \mathrm{I}$, and found the percentage of oxygen to be invariably 20.83 , a number nearly identical with those obtained by Bunsen and Regnault with much more perfect means. But the average chemist of that day obtained the most discordant results with the same apparatus and materials, and would doubtless also do so at the present day. By improved apparatus and methods the work of the average chemist is made to equal, or nearly so, that of the most skilful.

Volta introduced a new reagent-hydrogen-for the determination of oxygen, and he was the first to employ the electric spark in eudiometry. The use of mercury instead of water for confining the gases eliminated, the source of fallacy caused by transfusion through the latter liquid, and lastly, Bunsen, in the year I 839 , brought Volta's eudiometer to its highest degree of pertection.

The President then proceeded to describe and criticise the various forms of apparatus for the analysis of gaseous mixtures, and concluded as follows:--

Such are the modern developments of the eudiometer now at the disposal of chemists. For rapidity of working and delicacy of measurement they leave nothing to be desired; indeed, as regards delicacy, it may be doubted whether amongst all the instruments for measurement in this exhibition, there is one which can, like some of these eudiometers, give a distinct value in weight or volume to the one-fourteen-millionth part of a gramme of matter. Their drawback is their iragility, and any modifications to diminish this would doubtless be welcomed by chemists, since, chiefty for these reasons, eudiometry is still very rarely practised in chemical laboratories,

\section{THE PRESS ON THE LOAN COLLECTION}

$\mathrm{I}$ continuation of our article in last week's number we proceed to give a few more selections from the principal organs of public opinion, indicative of the light in which they regard the scientific collection which has been brought together at South Kensington. Last week we confined ourselves mainly to the daily press; this week we are able to cull the opinions of the principal weekly papers. Public opinion as thus expressed, it will be seen, all but unanimously approves of the collection as creditable to its organizers and to the country at large, as beneficial to the progress of science, and as calculated to have an important educative influence on the British public. We think the collection of public opinion as thus expressed will serve a good purpose. It will show to those men of science who have been more or less connected with the 
organization of the Loan Collection that their efforts have met with the approval of the intelligent and unprejudiced portion of their non-scientific fellow-countrymen, that these efforts have been unexpectedly successful, and that public opinion points to a perinanent successor as the natural outcome of this temporary collection.

The Saturday Review seldom gives way to unmeasured approval of any human effort; it is therefore extremely gratifying to find so severe a critic having nothing but praise to bestow on the collection. The following are a few extracts from last Saturday's number:-

"Mr. Spottiswoode, in his address as President of the first of the Conferences which have been arranged in connection with the Loan Collection of Scientific Apparatus at South Kensington, said that he was disposed to regard this Exhibition as marking an epoch in the history of science ; and there are undoubtedly reasons why it may be expected to exercise a deep and beneficial influence on the prospects of scientific culture in this country. We have here brought together, not only a collection of re. markable instruments from all parts of the civilized world, and representing almost every srhool and period of research, but also a numerous gathering of the men who are at the present moment engaged in extending still further the range of discovery, and the practical application of its results. It has often been a reproach a gainst this country on the part of foreigners that it is indifferent to science except in the forms in which it can be turned to immediate commercial profit ; and this criticism, though unjust to the heroic self-sacrifice which has characterised many of our leading scientific pioneers, must be admitted to be in a certain degree true as to the general attitude of the public. .... In this country the Executive usually hesitates to do anything unless there is a strong pressure of opinion, and it is tolerably certain that science will have little to hope for from that quarter until it has the public at its back ; and it is to it, therefore, that an appeal should be made. It may be hoped that the present Exhibition will be the beginning of a movement of this kind. The fact that it is opened under the auspices of a Government department would seem to show that there is not wanting a certain sympathy on that side; but whether any large; substantial measures will ever be taken, will chiefly depend on the interest which such a presentation of science excites among the community at large. Again, an Exhibition of this kind is useful in bringing to light the actual operations of the scientific world, the problems which have been solved, and those others which are still in a nebulous condition, with just here and there a clue peeping out; and thus the interchange of ideas is promoted.

At present this sort of co operation is loose, fraymentary, and disjointed; but an Exhibition brings the scaltered experimentalists into systematic communication. Thus, both in the world of science proper and outside of it, a keener interest is likely to be cultivated in regard to scientific matters, and researches will consequently be conducted with greater spirit and efficiency, and better prospects of success. . . . To persons of scientific training, or with even a rudimentary taste for such things, it is easy to conceive what service such an Exhibition will render. They will read the Handbook, an admirable summary of the chief branches of scientific stuciy by competent authorities, and examine the objects exhibited; and thus lay up a store of suggestive information as a supplement to or a foundation for private studies. But there will also be a large body of people who will chiefly bring away from the galleries an impression of their own stupendous ignor ance in such matters. This in itself, however, vill be a good thing, for it may be expected, in some cases at least, to stimulate a desire to know something, and aiter that to know more. Even the dullest and least imaginative minds can hardly fail to be touched by the sight of the instrumer:ts by which the old masters achieved their triumphs, or of their earliest works. . . . . On the other hand, this
Exhibition displays in a striking manner the wealth and luxury of scientific apparatus at the present day."

After giving examples of the intimate connection which subsists between the progress of science and the improvement of its mechanism, the article concludes thus :--

"It is impossible here to go through such an Exhibition in detail, and we can only say that it reflects much credit on those with whom it has originated, and that it is to be hoped that it may not be a mere passing show, but may develop into some permanent organization."

The Academy of last Saturday has " a first or introductory notice" of some length on the collection.

"The Special Loan Collection of Scientific Apparatus," the Academy says, "which was honoured by a private visit from her Majesty on Saturday last, and thrown open to the public on Monday, is one of very great interest and value. The Lord President of the Council may well be congratulated on the success of the undertaking, and we must all feel grateful to him for having given us an exhibition in which, for once, purely commercial interests have been made to give way to the "higher aim of disseminating as widely as possible a knowledye of the different methods of science.' The Exhibition is in many respects the most instructive and remarkable that has been held at South Kensington, and though it may not have any great effect on the advancement of science or on the industrial progress of this country, it cannot fail to awaken a very general interest in those methods of abstract scientific research of which the public know so little; and it will afford an opportunity, which may never occur again, of examining at leisure under the same roof the rude, simple instruments used by the pioneers of science, and the com. plex, delicate apparatus with which investigators of the present day have made their discoveries. We trust, too, that the Exhibition may give an impulse to the cause of scientific education in this country, and that it may lead to a better appreciation of the reasons which have led men of science to advocate Government endowment of scientific research, and the establishment of Physical Observatories, at home and abroad, which may have the same beneficial infuence on the progress of other sciences that Astronomical Observatories have had on the progress of astronomy. May we hope, too, that the Exhibition will lead to the creation of a museum for the illustration of physical, chemical, and mechanical sciences somewhat of the nature of the 'Conservatoire des Arts et Metiers,' in Paris? The formation of such a museun was one of the recommendations of the Commission on Scientific Instruction, and we believe it would go far, by affording adequate opportunities for study, to render the sciences alluded to as popular as those of botany, geology, and zoology."

Last week we quoted the opinion of Iron; the same paper has another interesting article this week, on "Science at South Kensirgton," in which it says that the success of the Exhibition affords an additional instance of the certain, if tardy, fructification of a valuable idea. "Years ago the conception of a great focus of science somewhere in the metropolis was formed in at least one great mind." The article then refers to the original intention of making the Albert Hall an institutional memorial, its employment as a place of scientific meetings and conferences having been strongly advocated. With its present uses, "the building has lost all its signification, as its position at South Kensington has lost all its appropriateness. We therefore cordially welcome the realisation of the spiritual part of the original plan, although it has been brought about by indirect means." "The article then goes on to refer to the successful development of loan collections during the last few years, and the superior educational value possessed by special collections over large international exhibitions. As carried out at South Kensington, this value is largely 
owing to classification, " a point kept distinc ily in view in arranging the Exhibition of Scientific Apparatus." The article then proceeds :-

"The problem of classification has been triumphantly solved. .... Success is absolute and complete.

"The institution of conferences during the Exhibition can hardly be regarded as other than a most valuable innovation, and precisely what was wanted-not to popularise the Exhibition, but to give it that life and movement without which the best institutions are apt to become stagnant, and be passed heedlessly by in an age of hurry and bustle. . . . There is no slackness at South Kensington, and conferences form an interesting and important part of the programme of the Scientific Exhibition which it is rumoured will probably prove the nucleus of a Scientific Museum analogous to the Conservatoire des Arts et Métiers at Paris. It would certainly be shortsighted policy to allow the splendid collection of objects now brought together for the first time in the world's history to be redistributed-scattered all over Europe, in odds and ends which teach little or nothing apart, but are of inestimable value when together. The want of a permanent national institution devoted to science can now be supplied in the least costly and most efficacious manner, that is to say, the vital part composed of the scientists and their instruments. As for the showy part--the outward and visible sign-the Central Hall of Science, it will come in time. If Albert Hall, after having failed as a music-hall, fails also as a circus and as a skating-rink, the country may one day be able to buy it up cheap, and convert it to a legitimate use."

The British Fournal of Photography says:-

"There is now open in the Exhibition Buildings, South Kensington, London, a large, varied, and most valuable collection of scientific apparatus and appliances. Its intrinsic value is great, its historical value much greater, but in its educational importance is to be found the chief value of this unique collection."

The Gardeners Chronicle speaks thus :-

"The splendid collections of Scientific Apparatus now on view at South Kensington may not have any great interest or attraction for the general public, for whose taste the display is too technical and unintelligible. To the more thoughtful visitor, and especially to the student, the collection is rich in interest and suggestiveness. . . The whole thing has been organised and got together so quietly that even among seientific men little or nothing was known about the proceedings till the list moment, and the extent and value of the collections has come upon them as a surprise."

Public opinion thus far, it will be seen, has nothing but admiration for the Loan Collection. The Athencum is on the other side. We give its article without note or comment, as the collection can hold its own.

"The galleries containing the Loan Collection of Scientific Instruments are at length open to the public. Apparently no expenditure has been considered too great by those who have been engaged in bringing together in the course of a few weeks from every part of Europe all the relics of science that could be begged or borrowed from public institutions or private collections. Gentlemen have been sent on special missions from South Kensing ton, and their movements have been duly chronicled in Reuter's telegrams amongst the most important news from Italy and Germany. Where these gentlemen could not find time to go, ambassadors and their attachés have been piessed into the service of collecting. Special railway trains have, we are informed by our contemporary, NATURE, been built for the transit of instruments, and the result is a collection of brass, glass, and old iron relics, which has driven the daily press wild with enthusiasm.

"According to the ordinary law of chances, a certain proportion of these instruments will be returned to the places whence they came all the worse for their journey across Europe, and we feel inclined to inquire whether it is certain that the worker in science will be the wiser for having seen them. The old and celebrated instruments have been repeatedly described and figured, and the new instruments, if useful, a man engaged in scientific research knows better than he knows the way to South Kensington. As to the curiosity-loving public, it will surely not be pretended that it is worth while to form such a collection for its amusement, but if it be the duty of government to gratify the craving of idlers, let us by all means at once appoint a Barnum to be Minister of Science; he will know how to make such exhibitions as this, and the School of Art needlework, a commercial success. But, no doubt, real instruction is intended, and if so, let us stop and ask whether the present is the best and cheapest plan of obtaining our object. The 'general public,' so far as can be judged from the experience of the first few days, regards the whole affair with indifference.

"In order to afford the means for studying the history of a science there is needed a continuous series of objects that will illustrate the development of thought step by step ; such a collection cannot be biought together in a few weeks. It needs the patient labour and study of a lifetime devoted to it; but in this exhibition, as in collections made by the nouveaux riches, the extremely old and extremely curious have been brought side by side with the complicated results of modern workmanship; and we find none of the connecting links, to gather which requires a man well versed in the history of his subject, and the labour of a lifetime. . . In fact, the collection required the control of a hand familiar with the history of astronomy. Objects that would have illustrated the development of the telescope during the seventeentil and eighteenth centuries should have been sought after more diligently than relics connected with great and popular names with which every one is familior.

"The general 'Handbook to the Exhibition,' which has been published, is a remarkably good shilling's-worth of information, but, as might be expected, it contains treatises of very different merit. After some general considerations on instruments by Prof. Clerk Maxwell, which will possibly be above the heads of most of his readers, follow some interesting though rather general disquisitions on various subjects, which have evidently in most cases been written without reference to the instruments brought together. The names of Prof. Clerk Maxwell, Prof. Smith, Prof, Clifford, Mr. Spottiswoode, Prof. Tait, and others, will be a sufficient guarantee of the trustworthiness of the information given. The article on Astronomy is not equal to the others, and considering the opportunity that the author had of illustrating the history of his subject, it is particularly poor and superficial. The 'Handbook' in general will well repay more than a casual perusal."

We did not state that "special railway trains" had been built for the purpose referred to above.

\section{NOTES}

THE Challenger is expected home daily, and arrangements are being made for the ship being weicomed on its arrival at Sheerness by the Royal Society and the foreign men of science now in this country.

THE visitation of the Royal Observatory is fixed for Saturday, June 3 .

The Anniversary Meeting of the Royal Geographical Society was held on Monday. The total number of ordinary Fellows on the list at the end of April was 3, 125. Sir H. Rawlinson, the President, presented the Founder's Medal to Lieut. Cameren, and the Patron's Medal to Mr. Lowther for Mr. J. Forrest, the Australian explorer. The annual geographical medals offered by the Society to the chief public schools vere prescnted to the 